PROCEEDINGS OF THE

AMERICAN MATHEMATICAL SOCIETY

Volume 103, Number 1, May 1988

\title{
EMBEDDING OF $U_{\omega}$-GROUPS IN $D_{\omega}$-GROUPS
}

\author{
CHARLES CASSIDY
}

(Communicated by Bhama Srinivasan)

\begin{abstract}
The purpose of this paper is to give certain new conditions under which it is possible to show that a group in which the equation $x^{p}=a$ has at most one solution for every $a$, can be embedded in another group in which the equation $x^{p}=a$ has exactly one solution for every $a$.

It has been known for a long time that locally nilpotent groups satisfy the above property but some other sufficient conditions have also been found by G. Baumslag in his thesis. Conditions related to Baumslag's are examined.
\end{abstract}

1. Introduction. In his fundamental paper [2], Baumslag studies three classes of groups associated with any set $\omega$ of prime numbers:

(1) $G$ is a $U_{\omega}$-group if when $p \in \omega, x, y \in G$ and $x^{p}=y^{p}$, then $x=y$;

(2) $G$ is an $E_{\omega}$-group if when $p \in \omega, y \in G$, then there exists $x \in G$ such that $x^{p}=y$;

(3) $G$ is a $D_{\omega}$-group if it is both a $U_{\omega}$-group and an $E_{\omega}$-group.

An obvious necessary condition for a group $G$ to be embedded in a $D_{\omega}$-group is that $G$ be a $U_{\omega}$-group. Although every group can be embedded in an $E_{\omega}$-group [4], it does not suffice that $G$ be a $U_{\omega}$-group for it to be embeddable in a $D_{\omega}$-group (see Neumann's counterexample in [1]).

For locally nilpotent groups, the above condition is known to be sufficient; this follows, for example, from the fact that the kernel of the $\pi$-localization map of $G$ is then $T_{\pi}(G)$, the $\pi^{\prime}$-torsion subgroup of $G$ where $\pi^{\prime}$ is the set of primes not belonging to $\pi$ (see [3]).

For groups that are not locally nilpotent, the localization is still not known in a sufficiently explicit manner to give much information on the above embedding problem (see, however, [6]). In that case, the only known set of sufficient conditions for a $U_{\omega}$-group $G$ to be embeddable in a $D_{\omega}$-group seems to be Baumslag's conditions [2] namely:

(a) If $p \in \omega$ and if $g \in G$ has no $p$ th root, then the centralizer $C(g, G)$ of $g$ in $G$ is isomorphic to a subgroup of $\Gamma_{\omega}=\{m / n ; m, n \in \mathbf{Z}$ and the only prime factors of $n$ are in $\omega\}$.

(b) If $p \in \omega$ and if $g \in G$ has no $p$ th root, then $C(g, G)=C\left(g^{m}, G\right)$ for all $m \in \mathbf{Z}$ $(m \neq 0)$.

(c) If $p \in \omega$, if $g \in G$ has no $p$ th root, and if $h^{-1} g^{m} h=g^{n}$ for some $h \in G$ and some integers $m$ and $n$, then $m=n$.

However, in the proof of his result, Baumslag uses, among several other things, his Lemma 28.7 in the proof of which there appears to be a mistake. Specifically,

Received by the editors February 20, 1986 and, in revised form, February 7, 1987.

1980 Mathematics Subject Classification (1985 Revision). Primary 20 E99.

The author gratefully acknowledges the assistance of the Canadian NSERC (A3097). 
on page 265, line 5, Baumslag claims: "Consequently $t_{1} \in G$ and $t_{n \mu} \in P$ ". One should observe by looking at his proof that this can only be true if, within the proof, $m$ and $n$ are both positive or both negative. Fortunately, as we shall point out later, one can overcome the difficulty by imposing the following additional requirement that:

(d) $G$ has no element of order 2 .

This extra condition imposes an additional constraint only when 2 does not belong to $\omega$ since otherwise a $U_{\omega}$-group does not have elements of order 2 . In this paper, we shall prove that any $U_{\omega}$-group $G$ can be embedded in a $D_{\omega}$-group provided it satisfies:

$\left(\mathrm{H}_{1}\right)$ If $p \in \omega$ and if $g \in G$ has no $p$ th root, then $C(g, G)$ is malnormal in $G$; that is, if $x \notin C(g, G)$, then $x C(g, G) x^{-1} \cap C(g, G)=\{1\}$.

$\left(\mathrm{H}_{2}\right) G$ has no element of order 2 .

These conditions are closely related to (a), (b), (c) and (d) since, on one hand, $\left(\mathrm{H}_{2}\right)$ and (d) are the same, and, on the other hand, (a), (b) and (c) imply $\left(\mathrm{H}_{1}\right)$ (Lemma 1) whereas $\left(\mathrm{H}_{1}\right)$ implies (b) and (c) (Lemma 2). Furthermore, any abelian group which is $U_{\omega}$ and has no element of order 2 automatically satisfies $\left(\mathrm{H}_{1}\right)$ and $\left(\mathrm{H}_{2}\right)$ although it does not satisfy (a) unless it is a subgroup of $\mathbf{Q}$; the same thing is true for free products of such groups. Our situation is thus more general than Baumslag's, but most steps of his proof can be used in our setting provided they are suitably interpreted. We shall, therefore, choose our notation so that we can refer to his proof as much as possible and focus on the differences, in particular the replacement of his Lemma 28.7 by our Proposition 2 .

\section{Preliminaries.}

LEMMA 1. Let $G$ be a $U_{\omega}$-group satisfying (a), (b) and (c). Then $G$ satisfies $\left(\mathrm{H}_{1}\right)$.

ProOF. Let $g \in G$ be an element not having all its $p$ th roots for $p \in \omega$. By (a), one sees that every $h \in C(g, G)$ satisfies $h^{m}=g^{n}$ for some $m, n \in \mathbf{Z}$. Notice that if we would have allowed $h=1$, then we would have had $g^{n}=1$ which is here impossible since, in a $U_{\omega}$-group, no prime factor of $n$ can be in $\omega$, but then $g$ clearly has all its $p$ th roots for $p \in \omega$. Let us assume that $x \notin C(g, G)$; if there exists $a \in x C(g, G) x^{-1} \cap C(g, G)$ with $a \neq 1$, then $a^{m_{1}}=g^{n_{1}}$ and $a^{m_{2}}=x g^{n_{2}} x^{-1}$ for some $m_{1}, n_{1}, m_{2}, n_{2} \in \mathbf{Z}$. It follows that

$$
g^{n_{1} m_{2}}=a^{m_{1} m_{2}}=x g^{n_{2} m_{1}} x^{-1},
$$

and, using (c), we have $n_{1} m_{2}=n_{2} m_{1}$; hence, $x \in C\left(g^{n_{1} m_{2}}, G\right)$, which is also $C(g, G)$ by (b), but this is impossible since we have assumed that $x \notin C(g, G)$.

LEMMA 2. Let $G$ be a $U_{\omega}$-group satisfying $\left(\mathrm{H}_{1}\right)$. Then $G$ satisfies $(\mathrm{b})$ and $(\mathrm{c})$.

ProOF. Let $g \in G$ be an element not having all its $p$ th roots for $p \in \omega$.

One has always $C(g, G) \leq C\left(g^{m}, G\right)$. On the other hand, if $a \in C\left(g^{m}, G\right)$, then $a^{-1} g^{m} a=g^{m}$ means that both $g^{m}$ and $a^{-1} g^{m} a$ belong to $C(g, G)$, and since $G$ satisfies $\left(\mathrm{H}_{1}\right)$, then $a \in C(g, G)$ and (b) is satisfied.

If now $h^{-1} g^{m} h=g^{n}$ for some $h \in G$, then by $\left(\mathrm{H}_{1}\right)$ we have $h \in C(g, G)$. It follows that $h^{-1} g^{m} h=g^{m}$. Since in a $U_{\omega}$-group, an element $g$ not having all its $p$ th roots must be of infinite order, it follows that $m=n$, and (c) is satisfied. 
A trivial but interesting property of $U_{\omega}$-groups will play an essential role in our construction. Let $G$ be a $U_{\omega}$-group and let $g \in G$ be any element of infinite order. We denote by $R_{\omega}(g, G)$ the set of all $g^{m / n}$, where $m, n \in \mathbf{Z}$ and the only prime factors of $n$ are in $\omega$. Notice that if $g=h^{n}$ with $n \notin \omega$, then $h \notin R_{\omega}(g, G)$. We also note that $g^{m / n}$, when it exists, can designate only one element of $G$ since $G$ is $U_{\omega}$. Then $R_{\omega}(g, G)$ is a central subgroup of $C(g, G)$ which is isomorphic to a subgroup of $\Gamma_{\omega}$. (This is essentially trivial since it suffices to show that $g^{m_{1} / n_{1}} g^{m_{2} / n_{2}}=$ $g^{\left(m_{1} n_{2}+m_{2} n_{1}\right) / n_{1} n_{2}}$ but this follows at once from the fact that, in a $U_{\omega}$-group, an element $g$ and all its $p$ th roots $(p \in \omega)$ must commute with the same elements).

It is thus possible to form the central product $P_{\omega}(g, G)$ of $C(g, G)$ and $\Gamma_{\omega}$ over $R_{\omega}(g, G)$ where we agree to consider $R_{\omega}(g, G)$ as a subgroup of $\Gamma_{\omega}$ through the homomorphism $i_{2}$ that sends $g$ to 1 ; then $P_{\omega}(g, G)$ is the quotient of the cartesian product $C(g, G) \times \Gamma_{\omega}$ by the normal subgroup $D=\left\{\left(i_{1}(x), i_{2}\left(x^{-1}\right)\right) \mid x \in R_{\omega}(g, G)\right\}$. One remarks that $C(g, G), \Gamma_{\omega}$, and $R_{\omega}(g, G)$ can all be seen as subgroups of $P_{\omega}(g, G)$, that $g$ has all its $p$ th roots in $P_{\omega}(g, G)$ for $p \in \omega$ and that $\Gamma_{\omega}$ is central in $P_{\omega}(g, G)$.

From now on we shall consider that $G, g$, and $\omega$ are fixed and we shall write, whenever no confusion arises, $Z$ for $C(g, G), R$ for $R_{\omega}(g, G), P$ for $P_{\omega}(g, G)$, and $\Gamma$ for $\Gamma_{\omega}$.

LEMMA 3. Let $H$ and $K$ be arbitrary $U_{\omega}$-groups. Suppose that the abelian group $A$ can be seen as a central subgroup of both $H$ and $K$ and suppose furthermore that $A$ is $\omega$-isolated in one of them, say $H$. Then the central product of $H$ and $K$ over $A$ is also a $U_{\omega}$-group. In particular, with the above notation, $P$ is a $U_{\omega}$-group.

ProOF. Suppose that $\left(\overline{h_{1}, k_{1}}\right)^{p}=\left(\overline{h_{2}, k_{2}}\right)^{p}$ where $h_{1}, h_{2} \in H, k_{1}, k_{2} \in K$, and $p \in \omega$. Then $\left(\overline{h_{1}^{p}, k_{1}^{p}}\right)=\left(\overline{h_{2}^{p}, k_{2}^{p}}\right)$ and it follows that $h_{2}^{-p} h_{1}^{p} \in A$ which is central in $H$ and then $h_{1}^{p}$ and $h_{2}^{p}$ commute. The elements $h_{1}$ and $h_{2}$ also commute since they both belong to the $U_{\omega}$-group $G$. The same argument applies to $k_{1}$ and $k_{2}$ which thus also commute. One has $\left(h_{2}^{-1} h_{1}\right)^{p}=h_{2}^{-p} h_{1}^{p}=k_{2}^{p} k_{1}^{-p}=\left(k_{2} k_{1}^{-1}\right)^{p}$. The problem would be that $h_{2}^{-1} h_{1}$ and $k_{2} k_{1}^{-1}$ could belong to different groups but our assumption that $A$ is isolated in $H$ makes it clear that they both belong to $K$ and are thus equal since $K$ is $U_{\omega}$. It then follows that $\left(\overline{h_{1}, k_{1}}\right)=\left(\overline{h_{2}, k_{2}}\right)$ and the proof is completed.

The last step in our construction consists in forming the generalised free product $F$ of $G$ and $P$ (with $Z$ amalgamated), that is, $G * z P$.

We shall show that if $G$ is a $U_{\omega}$-group satisfying $\left(\mathrm{H}_{1}\right)$ and $\left(\mathrm{H}_{2}\right)$, then $F$ is again a $U_{\omega}$-group satisfying $\left(\mathrm{H}_{1}\right)$ and $\left(\mathrm{H}_{2}\right)$. The difference will be that $g$ will have all its $p$ th roots in $F$ (for $p \in \omega$ ).

\section{The proof.}

LEMMA 4. If $G$ is a $U_{\omega}$-group satisfying $\left(\mathrm{H}_{2}\right)$, then $F$ satisfies $\left(\mathrm{H}_{2}\right)$.

ProOF. We first show that $P$ satisfies $\left(\mathrm{H}_{2}\right)$. When $2 \in \omega$, this is a consequence of Lemma 3. Let us thus suppose that $2 \notin \omega$. Take $(\overline{h, y})$ in $P$ such that $(\overline{h, y})^{2}=1$, that is $h^{2}=y^{-2} \in R$; we see that $y^{-1} \in R$ since both $y^{-2} \in R$ and $y^{n} \in R$ for some odd $n \in \omega$. It follows that $(h y)^{2}=1$ holds in $G$ but since $G$ has no element of order 2 , then $h y=1$, that is $(\overline{h, y})=1$. It follows that $F$ cannot have any element 
of order 2 since such an element would have to be conjugate to either an element of $G$ or an element of $P$ by [2, Theorem 16.4].

PROPOSITION 1. If $G$ is a $U_{\omega}$-group satisfying $\left(\mathrm{H}_{1}\right)$, then $F$ is a $U_{\omega}$-group.

PROOF. We shall not give it since it is entirely similar to Baumslag's proof of the first part of Theorem 29.1 [2, p. 266].

Baumslag's proof rests upon a sequence of six lemmas (from Lemma 28.1 to Lemma 28.6) all of which, except Lemma 28.1, are also true in our situation and with the same proofs. The only part of Lemma 28.1 which remains true will be stated as our Lemma 5 . It is the only inclusion needed to prove the subsequent lemmas and Baumslag's proof of it remains valid here. The converse inclusion which was obvious in his situation is no longer true in our case (see however Lemma 8).

We must insist on the fact that our $P$, and hence our $F$, have different meanings from those in [2] but they play exactly the same roles and all the proofs in [2] mentioned above can be easily read taking note of these differences. We shall now state three of the above-mentioned lemmas which will also be needed in the proof of Proposition 2.

LeMmA 5. For every $a \in P(a \neq 1), C(a, F) \leq P$.

LEMMA 6. Let $a \in G$ and suppose $a$ is not conjugate to an element of $Z$. Then $C(a, F)=C(a, G)$.

LEMMA 7. Suppose that $a \in F$ is conjugate neither to an element of $G$ nor to an element of $P$. Then $C(a, F)$ is an infinite cyclic group. Moreover, if $a$ is cyclically reduced, every nontrivial $x$ in $C(a, F)$ is also cyclically reduced and is conjugate neither to an element of $G$ nor to an element of $P$.

We shall need also the following partial converse of Lemma 5.

LEMMA 8. If $G$ satisfies $\left(\mathrm{H}_{1}\right)$ and if $a \in P$ does not have all its pth roots $(p \in \omega)$, then $P \leq C(a, F)$.

Proof. Since $g \in \Gamma$ which is central in $P$, we certainly have $P \leq C(g, F)$. Let us take any $a \in P$ not having all its $p$ th roots. There exists $n$ (a product of primes in $\omega$ ) such that $a^{n} \in Z$. Similarly, for any $x \in P$, there exists $m$ (a product of primes in $\omega$ ) such that $x^{m} \in Z$. Now $x^{m}$ commutes with $a^{n}$ because condition $\left(\mathrm{H}_{1}\right)$, that also applies to $a^{n}$ (which does not have all its $p$ th roots), implies that $C\left(a^{n}, G\right)=C(g, G)=Z$. Since both $a$ and $x$ belong to the $U_{\omega}$-group $P$, then they must also commute because $m$ and $n$ are products of primes in $\omega$. We thus have $P \leq C(a, F)$.

Before the proof of the next key proposition, it is necessary to recall some basic facts and terminology concerning a generalised free product with amalgamation $F=G *_{Z} P$ (see [5]).

If we choose once and for all in $G$ a system $S_{G}$ of left coset representatives modulo $Z$ containing the unit element, then every element $g$ in $G$ can be written uniquely in the form $g=s z\left(s \in S_{G}, z \in Z\right)$. Similarly a system $S_{P}$ in $P$ gives a unique representation of every element in $P$ as $p=s z\left(s \in S_{P}, z \in Z\right)$. 
After having chosen the systems $S_{G}$ and $S_{P}$, we say that an element $w$ in $F$ is a normal word if $w=s_{1} s_{2} \cdots s_{n} z$, where:

(i) Every component $s_{i}(i \leq n)$ is a representative $\neq 1$ belonging to one of the $S_{G}$ or $S_{P}$.

(ii) Successive components $s_{i}$ and $s_{i+1}$ do not belong to the same system $S_{G}$ or $S_{P}$.

(iii) The last component $z$ belongs to the common subgroup $Z$.

We call $n$ the length of the normal word; it is denoted $\lambda(w)$. It is known that every element $f$ in $F$ is represented by one and only one normal word called the normal form of $f$. We say that $f \in F$ is cyclically reduced if none of its conjugates in $F$ has smaller length than $f$ itself. If $w$ is the normal form of $f$ and if $\lambda(w) \geq 2$, then $f$ is cyclically reduced if and only if $s_{1}$ and $s_{n}$ belong to different components, that is in our case if and only if $n$ is even.

We are now ready to state and prove the following

PROPOSITION 2. If $G$ is a $U_{\omega}$-group satisfying $\left(\mathrm{H}_{1}\right)$ and $\left(\mathrm{H}_{2}\right)$, then $F$ also satisfies $\left(\mathrm{H}_{1}\right)$.

PROOF. We must show that if $p \in \omega$ and if $a \in F$ does not have a $p$ th root in $F$ for some $p \in \omega$ then $h \in C(a, F)(h \neq 1)$ and $x^{-1} h x \in C(a, F)$ imply $x \in C(a, F)$.

We can assume that $a$ is cyclically reduced since otherwise $f^{-1} a f=b$ is cyclically reduced for some $f \in F$ and then $f^{-1} h f \in C(b, F)$ and $\left(f^{-1} x f\right)^{-1}\left(f^{-1} h f\right)\left(f^{-1} x f\right)$ $\in C(b, F)$ imply $f^{-1} x f \in C(b, F)$, that is $x \in C(a, F)$.

Furthermore, any element of finite order in $F$ must be conjugate to an element of either $G$ or $P$ by [2, Theorem 16.4], and since both $G$ and $P$ are $U_{\omega}$-groups, then such an element must have all its $p$ th roots $(p \in \omega)$; it thus follows that $a$ is of infinite order. Now the proof splits in several cases.

Suppose firstly that $a \in P$. Since $a$ does not have all its $p$ th roots, it follows from Lemmas 5 and 8 that $C(a, F)=P$. Let us write $x=c_{1} c_{2} \cdots c_{n}$ where no two successive factors $c_{i}, c_{i+1}$ are elements of the same group $G$ or $P$. We thus have $x^{-1} h x=c_{n}^{-1} \cdots c_{2}^{-1} c_{1}^{-1} h c_{1} c_{2} \cdots c_{n}$ and if $h \in P-Z$, then $x^{-1} h x$ can only belong to $P$ if $c_{1}$ belongs to $P$; if $n=1$, this means that $x \in P=C(a, F)$; if $n>1$, then we must have $c_{1}^{-1} h c_{1} \in Z$ and $c_{2}^{-1}\left(c_{1}^{-1} h c_{1}\right) c_{2} \in Z$ which contradicts $\left(\mathrm{H}_{1}\right)$ since $c_{2}$ belongs to $G-Z$. If now $h \in Z$ and $c_{1} \in G$, then $x^{-1} h x \in P$ implies $c_{1}^{-1} h c_{1} \in Z$ but then, by $\left(\mathrm{H}_{1}\right), c_{1} \in Z$ which, by our assumption on the $c_{i}$ 's is only possible if $n=1$ and then $x=c_{1} \in Z \leq C(a, F)$. If $h \in Z$ and $c_{1} \in P$, then if $n>1$, we cannot have $x^{-1} h x \in P$ since then we should have $c_{1}^{-1} h c_{1} \in Z$ and $c_{2}^{-1}\left(c_{1}^{-1} h c_{1}\right) c_{2} \in Z$ but this contradicts $\left(\mathrm{H}_{1}\right)$ for $c_{2} \in G-Z$. We thus have again $x=c_{1} \in P=C(a, F)$.

Suppose secondly that $a \in G-Z$. Since $a$ is not conjugate to an element in $Z$, then $C(a, F)=C(a, G)$ by Lemma 6 . Moreover, by $\left(\mathrm{H}_{1}\right), C(a, G)$ and $Z$ cannot have in common a nontrivial element $y$ (otherwise one would have both $y$ and $a^{-1} y a$ in $Z$, so that $a \in Z$, a contradiction). We can thus suppose that both $h$ and $x^{-1} h x$ are in $G-Z$ but this can only occur if $c_{1} \in G$. This is not possible if $n>1$ since we would then have $c_{1}^{-1} h c_{1} \in Z$ but since $h \in C(a, G)$ we would then have, by $\left(\mathrm{H}_{1}\right), c_{1}^{-1} a c_{1} \in Z$, which contradicts the hypotheses that $a$ is cyclically reduced. We must have $n=1$ and $x=c_{1} \in G$ but, by $\left(\mathrm{H}_{1}\right), x$ then belongs to $C(a, G)$. 
Suppose finally $\lambda(a) \geq 2$. Then, by Lemma 7 , both $h$ and $x^{-1} h x$ are also cyclically reduced of length $\geq 2$ and $C(h, F)=C\left(x^{-1} h x, F\right)=C(a, F) \approx \mathbf{Z}$. Suppose that $c$ is a generator of $C(a, F)$. Then $h=c^{\mu}, x^{-1} h x=c^{\nu}$ for some $\mu, \nu \in Z$; it follows that $\left(x^{-1} c x\right)^{\mu}=x^{-1} c^{\mu} x=c^{\nu}$, hence $x^{-1} c x$ commutes with $c^{\nu}$ and thus $x^{-1} c x$ can be written as $c^{\rho}$; we then have $c^{\rho \mu}=c^{\nu}$ so $\mu$ divides $\nu$. Similarly, by letting $x^{-1}$ operate on $c^{\nu}$ by conjugation, one sees that $\nu$ divides $\mu$. It does follow that $x^{-1} h x=h$ or $h^{-1}$. If $x^{-1} h x=h$, then $x \in C(h, F)=C(a, F)$ and it is what we wanted to prove. On the other hand, $x^{-1} h x=h^{-1}$ is impossible for then one would have $x^{-2} h x^{2}=h$, hence $x^{2} \in C(h, F)$ but $x \notin C(h, F)$. If $x^{2}$ were a nontrivial element of $C(h, F)$, we should have $C\left(x^{2}, F\right)=C(x, F)$ as before by Lemma 7 . It follows that $x^{2}$ must be trivial but this is also impossible by Lemma 4. We thus have achieved the proof of the proposition.

Proposition 3. Let $G$ be a $U_{\omega}$-group satisfying $\left(\mathrm{H}_{1}\right)$ and $\left(\mathrm{H}_{2}\right)$ and let $g$ be any element of $G$. Then $G$ can be embedded in a $U_{\omega}$-group $\bar{G}$ in which $g$ has all its pth rocts $(p \in \omega)$; moreover, we can choose $\bar{G}$ so that it satisfies $\left(\mathrm{H}_{1}\right)$ and $\left(\mathrm{H}_{2}\right)$.

Proof. If $g$ has all its $p$ th roots in $G$, we take $\bar{G}=G$. Otherwise, we use the construction described above and the result follows directly from Lemma 4, Proposition 1 and Proposition 2.

THEOREM 1. Let $G$ be a $U_{\omega}$-group satisfying $\left(\mathrm{H}_{1}\right)$ and $\left(\mathrm{H}_{2}\right)$. Then $G$ can be embedded in a $D_{\omega}$-group.

ProOF. One uses the classical ascending tower argument as in [2, pp. 268-270]. The idea is to embed firstly $G$ in a $U_{\omega}$-group $\bar{G}$ having $p$ th roots for all the elements of $G$ and satisfying $\left(\mathrm{H}_{1}\right)$ and $\left(\mathrm{H}_{2}\right)$. This can be done by considering a well-order of the elements of $G$ and then using repeatedly Proposition 3 to adjoin the $p$ th roots while preserving $\left(\mathrm{H}_{1}\right),\left(\mathrm{H}_{2}\right)$ and the property of being a $U_{\omega}$-group. We must also observe that these three properties are also preserved by taking ascending unions.

Secondly, we put $G_{0}=G$ and define, inductively, $G_{i+1}=\bar{G}_{i}$. The union, for $i \in \mathbf{N}$, of the ascending sequence of the $G_{i}$ 's is then clearly a $D_{\omega}$-group containing $G$.

\section{BIBLIOGRAPHY}

1. G. Baumslag, Wreath products and p-groups, Proc. Cambridge Philos. Soc. 55 (1959), 224-231.

2. ___ Some aspects of groups with unique roots, Acta Math. 104 (1960), 217-303.

3. P. Hilton, On direct limits of nilpotent groups, Lecture Notes in Math., vol. 418, SpringerVerlag, Berlin, Heidelberg and New York, 1974, pp. 68-77.

4. B. H. Neumann, Adjunction of elements to groups, J. London Math. Soc. 18 (1943), 4-11.

5. _ An essay on free products of groups with amalgamations, Philos. Trans. Roy. Soc. London Ser. A 246 1954, 503-554.

6. P. Ribenboim, Equations in groups, with special emphasis on localization and torsion, Queen's Preprint 1982-22, Kingston, Ontario, Canada.

Département de Mathématiques et de Statistique, Université laval, QUÉBEC, CANADA 